\title{
SHEARING MOTIONS AND THE FORMATION OF SHOCKS IN AN ELASTIC CIRCULAR TUBE*
}

\author{
BY \\ R. L. FOSDICK AND G. P. MACSITHIGH \\ University of Minnesota
}

1. Introduction. It is typical of nonlinear hyperbolic equations $\dagger$ in two variables that discontinuities may develop in a solution which emanates from smooth initial data. The oldest and best known example of this phenomenon is the piston problem of onedimensional gas dynamics. Whereas expansive motions give rise to a globally smooth solution, compression yields one for which continuity is preserved, but for which smoothness breaks down in a finite time. Most of the known results of this type concern $2 \times 2$ systems. Zabusky [6] has shown that a model equation for a nonlinear elastic string will exhibit a shock discontinuity in finite time for a specific choice of smooth initial data. More generally Lax [4] has discussed this problem for a general homogeneous $2 \times 2$ system, and, subject to an assumption of universal genuine nonlinearity, has solved it completely. His main result may be summarized as follows: Let the system

$$
\frac{\partial y}{\partial t}+\lambda(y, z) \frac{\partial y}{\partial x}=0, \quad \frac{\partial z}{\partial t}+\mu(y, z) \frac{\partial z}{\partial x}=0
$$

be universally genuinely nonlinear; i.e. for example, let $\lambda_{y}(y, z) \leq-m_{1}<0$, and $\mu_{z}(y, z) \leq$ $-m_{2}<0$, for all $y$ and $z$. Let $[y(x, t), z(x, t)]$ be a solution of $(1.1)$ whose initial values are bounded. Then, (i) if $y_{x}(x, 0)>0$ or if $z_{x}(x, 0)>0$ at any point $x$, the first partial derivatives of the solution become unbounded after finite time, or (ii) if $y(x, 0)$ and $z(x, 0)$ are nonincreasing functions of $x$, the first partial derivatives of the solution remain uniformly bounded and the solution exists and is differentiable for all $t>0$. He obtained similar results for each of the other natural cases of universal genuine nonlinearity. Lax's method of analysis was to generate ordinary differential equations for the evolution along characteristic curves of the quantities $y_{x}(x, t), z_{x}(x, t)$ and to obtain estimates for their solutions.

Rectilinear motion of an incompressible, isotropic, elastic body gives rise to the dynamical equation

$$
Q^{2}\left(w_{x}\right) w_{x x}=\rho w_{t t}
$$

\footnotetext{
* Received September 10, 1979; revised version received December 3, 1979. The authors gratefully acknowledge the support of the National Science Foundation.

$\dagger$ Throughout this work, we shall, without further comment, draw repeatedly on the standard notations and terminologies of two fields, the theory of finite elasticity and that of nonlinear hyperbolic systems. An excellent account of the former subject is given in [18] and thorough treatments of the latter may be found in, for example, [3], [5], or [9].
} 
which, in terms of Riemann invariants, has the form

$$
\frac{\partial y}{\partial t}-\bar{\Gamma}(y-z) \frac{\partial y}{\partial x}=0, \quad \frac{\partial z}{\partial t}+\bar{\Gamma}(y-z) \frac{\partial z}{\partial x}=0 .
$$

While this system is not universally genuinely nonlinear for many functions $\bar{\Gamma}(\cdot)$ which arise naturally in the constitutive theory of elastic solids, MacCamy and Mizel [7], nevertheless, have addressed this problem by means of the hodograph transformation, for a class of functions $Q(\cdot)$ which are indeed compatible with elasticity theory. They found that for almost all choices of convex initial data, the solution must develop a shock discontinuity.

While the approach of Lax [4] has been somewhat refined by Jeffrey [8], Chang [15] has pursued the method of MacCamy and Mizel with some relaxation of the conditions on $Q(\cdot)$.

Recently, the inhomogeneous system

$$
\frac{\partial y}{\partial t}-\bar{\Gamma}(y-z) \frac{\partial y}{\partial x}=-\frac{\alpha}{2}(y+z), \quad \frac{\partial z}{\partial t}+\bar{\Gamma}(y-z) \frac{\partial z}{\partial x}=-\frac{\alpha}{2}(y+z),
$$

where the constant $\alpha$ is positive, has been studied by Nishida [10] and Slemrod [11]. Nishida has shown that the initial value problem for (1.4) will possess a unique smooth solution for $t>0$, provided that $\bar{\Gamma}(0)>0$ and that $y(x, 0), z(x, 0), y_{x}(x, 0)$, and $z_{x}(x, 0)$ are chosen sufficiently small. Slemrod, on the other hand, has shown that if $y(x, 0)$ and $z(x, 0)$ are sufficiently small and $\bar{\Gamma}(0)>0, \bar{\Gamma}^{\prime}(0)<0$, then if $y_{x}(x, 0)$ or $z_{x}(x, 0)$ is positive and sufficiently large at any point $x \in \mathbb{R}$, the initial-value problem for (1.4) has a solution in $C^{1}\left(\mathbb{R}^{2}\right)$ only for a finite time. He finds a similar result for the case $\bar{\Gamma}^{\prime}(0)>0$, provided $y_{x}(x, 0)$ or $z_{x}(x, 0)$ is negative and sufficiently large at any point $x \in \mathbb{R}$.

Rozhdestvenskii [12] has examined the initial-value problem for the general inhomogeneous $2 \times 2$ system

$$
\frac{\partial y}{\partial t}+\xi_{1}(y, z, x, t) \frac{\partial y}{\partial x}=f_{1}(y, z, x, t), \quad \frac{\partial z}{\partial t}+\xi_{2}(y, z, x, t) \frac{\partial z}{\partial x}=f_{2}(y, z, x, t) .
$$

He found that a necessary and sufficient condition for globally smooth solutions to exist for all choices of smooth initial data is

$$
\xi_{1} \equiv \xi_{1}(z, x, t), \quad \xi_{2}=\xi_{2}(y, x, t),
$$

which he called the condition of weak nonlinearity. He proved necessity by constructing an example of initial data which led to a breakdown of smoothness provided (1.6) is violated.

John [13] has considered the homogeneous $n \times n$ system

$$
\mathbf{u}_{t}+\mathbf{A}(\mathbf{u}) \mathbf{u}_{x}=0
$$

and has shown, by means of a Lax-type analysis, that simple wave solutions of (1.7) emanating from suitably chosen smooth initial data must lose differentiability after a finite time.

Kosinski [14] has investigated the inhomogeneous $n \times n$ system

$$
\mathbf{u}_{t}+\mathbf{A}(\mathbf{u}) \mathbf{u}_{x}=\mathbf{B}(\mathbf{u}) .
$$


Under rather drastic technical assumptions on the matrix $\mathbf{A}(\cdot)$ and on the vector $\mathbf{B}(\cdot)$, he obtained existence and non-existence results for arbitrary $n$, and, under somewhat milder conditions, he gave similar results for the case $n=2$.

When a breakdown in smoothness of the sort described in the preceding paragraphs occurs, the problem as originally posed must be reinterpreted and a generalized solution must be sought in a suitably extended class of functions. For problems in continuum physics a very natural such class is that consisting of piecewise smooth functions, since this class admits the possibility of shock discontinuities as purely "jumps." However, solutions of nonlinear hyperbolic problems which are piecewise smooth for all time in general do not exist; in fact, this is so even in the case of a single first-order conservation law. In this regard, whereas Schaeffer [20] has shown that the initial value problem for such an equation has a generically piecewise smooth solution, nevertheless there are examples of problems with $C^{\infty}$-initial data which do not lead to piecewise smooth solutions.

There exist many other articles on the breakdown of solutions of nonlinear hyperbolic systems. Among these, one which we have found both novel and interesting is that of Knops, Levine and Payne [19] in which they concentrate on breakdown of an entirely different nature from that connected with the formation of a shock discontinuity. They are concerned with conditions that lead to the existence of a "finite escape time." In terms of general nonlinear elastodynamics, they place what appear to be reasonably acceptable inequality restrictions on the strain energy function and show that in certain initial boundary-value problems the displacement vector itself must become unbounded in a finite time.

In the present work, we seek analogues to the results of Zabusky, Lax, and McCamy and Mizel for one-dimensional elastodynamic motions with circular symmetry. When expressed in terms of Riemann invariants, the initial boundary-value problem that we shall consider for the domain $r \in[a, b], b>a>0, t \geq 0$, is of the form

$$
\begin{array}{rlrl}
\frac{\partial y}{\partial t}-\bar{\Gamma}(y-z) \frac{\partial y}{\partial r}=\frac{1}{r} \pi_{1}(y, z), & & \frac{\partial z}{\partial t}+\bar{\Gamma}(y-z) \frac{\partial z}{\partial r}=\frac{1}{r} \pi_{2}(y, z), \\
y(r, 0) & =y_{0}(r), & & z(r, 0)=z_{0}(r), \\
y(a, t)+z(a, t) & =h_{1}(t), & & y(b, t)+z(b, t)=h_{2}(t) .
\end{array}
$$

This system has a number of disagreeable features. First, it is inhomogeneous, so that the Riemann invariants are not constant along their associated characteristics. Moreover, this fact also means that an application of the hodograph transformation yields no simplification. In addition, the present of the $1 / r$ factor denies us the customary device of transforming (for suitable boundary data) the initial boundary-value problem to an equivalent initial-value problem. Thus, we see that neither the approach of Lax nor that of MacCamy and Mizel may be applied to the present problem. We shall therefore employ the method of Rozhdestvenskii to study this problem in the domain of influence of the initial data, where it is, in effect, an initial-value problem. More specifically, we shall delineate somewhat extensive classes of initial data which lead to the breakdown of smoothness.

While it would be satisfying to exhibit examples of data for the initial-, boundaryvalue problem which yield globally smooth solutions, we recognize that even in the local 
existence question for such problems a solution with a particular degree of smoothness is contingent upon compatibility conditions of the same degree between initial and boundary data (see, for example, $[2,16]$ ). Thus, we shall not consider the existence of global smooth solutions in this work.

2. The dynamical equations. In the absence of body forces, the local dynamical equations for a continuum are given, in terms of the physical stress components $T\langle i j\rangle$ relative to a fixed cylindrical polar coordinate system, by

$$
\begin{gathered}
\left.\rho \mid \frac{\partial^{2} r}{\partial t^{2}}-r\left(\frac{\partial \theta}{\partial t}\right)^{2}\right\}=\frac{\partial}{\partial r} T\langle r r\rangle+\frac{1}{r} \frac{\partial}{\partial \theta} T\langle r \theta\rangle+\frac{1}{r}[T\langle r r\rangle-T\langle\theta \theta\rangle], \\
\rho\left\{r \frac{\partial^{2} \theta}{\partial t^{2}}+2 \frac{\partial r}{\partial t} \frac{\partial \theta}{\partial t}\right\}=\frac{1}{r^{2}} \frac{\partial}{\partial r}\left\{r^{2} T\langle r \theta\rangle\right\}+\frac{1}{r} \frac{\partial}{\partial \theta} T\langle\theta \theta\rangle+\frac{\partial}{\partial z} T\langle\theta z\rangle, \\
\rho \frac{\partial^{2} z}{\partial t^{2}}=\frac{1}{r} \frac{\partial}{\partial r}\{r T\langle r z\rangle\}+\frac{1}{r} \frac{\partial}{\partial \theta} T\langle\theta z\rangle+\frac{\partial}{\partial z} T\langle z z\rangle,
\end{gathered}
$$

where $(r, \theta, z)$ represents the instantaneous cylindrical coordinate of a material particle which is located at $(R, \Theta, Z)$ in a reference configuration.

For an isotropic, incompressible, elastic solid, the stress tensor $\mathbf{T}$ is related to the motion through the constitutive relation

$$
\mathbf{T}=-p \mathbf{1}+\alpha_{1}(I, I I) \mathbf{B}+\alpha_{-1}(I, I I) \mathbf{B}^{-1},
$$

where $\mathbf{B}$ is the left Cauchy-Green tensor and $I$ and $I I$ denote, respectively, its first and second principal invariants. The constraint of incompressibility requires that $\mathbf{B}$ must have unit determinant.

In this paper, we consider the following two types of motion regarding axial and annular shearing of tubes.

(i) Longitudinal shearing of an annular tube. This motion is given by

$$
r(R, \Theta, Z, t)=R, \quad \theta(R, \Theta, Z, t)=\Theta, \quad z(R, \Theta, Z, t)=Z+u(R, t),
$$

and the physical components, $B\langle i j\rangle$, of the left Cauchy-Green tensor are readily computed to be

$$
\mathbf{B}=\left(\begin{array}{ccc}
1 & 0 & u_{r} \\
0 & 1 & 0 \\
u_{r} & 0 & 1+u_{r}^{2}
\end{array}\right), \quad u_{r} \equiv \partial u / \partial r
$$

This motion is clearly isochoric, and it follows that the physical components of the associated stress tensor are given by

$$
\mathbf{T}=-p\left(\begin{array}{lll}
1 & 0 & 0 \\
0 & 1 & 0 \\
0 & 0 & 1
\end{array}\right)+\alpha_{1}(I, I I)\left(\begin{array}{ccc}
1 & 0 & u_{r} \\
0 & 1 & 0 \\
u_{r} & 0 & 1+u_{r}^{2}
\end{array}\right)+\alpha_{-1}(I, I I)\left(\begin{array}{ccc}
1+u_{r}^{2} & 0 & -u_{r} \\
0 & 1 & 0 \\
-u_{r} & 0 & 1
\end{array}\right)
$$

where

$$
I=I I=3+\left(u_{r}\right)^{2}
$$


From (2.1), by assuming $p$ to be a function of $r$ and $t$ only, we see that

$$
\begin{gathered}
\rho \frac{\partial^{2} u}{\partial t^{2}}=\frac{1}{r} \frac{\partial}{\partial r}\left\{r\left[\alpha_{1}(I, I I)-\alpha_{-1}(I, I I)\right] \frac{\partial u}{\partial r}\right\} \\
\frac{\partial}{\partial r} p(r, t)=\frac{\partial}{\partial r} \mid \alpha_{1}(I, I I)+\left\{1+\left(\frac{\partial u}{\partial r}\right)^{2} \mid \alpha_{-1}(I, I I)\right\}+\frac{1}{r} \alpha_{-1}(I, I I)\left(\frac{\partial u}{\partial r}\right)^{2} .
\end{gathered}
$$

Thus, upon solving the first of these equations for $u$, we may use the second to find the pressure $p$ which is needed to maintain the motion. Since we are concerned with the motion of an annulus for which $a$ and $b$ represent the inner and outer radius, respectively, the initial boundary-value problem which we pose for $u$ is of the following form:

$$
\rho \frac{\partial^{2} u}{\partial t^{2}}(r, t)=\frac{1}{r} \frac{\partial}{\partial r}\left\{r \tau\left(u_{r}\right) u_{r}\right\}, \quad 0<a \leq r \leq b, \quad 0 \leq t,
$$

with initial conditions

$$
u(r, 0)=f_{1}(r), \quad u_{t}(r, 0)=f_{2}(r), \quad a \leq r \leq b,
$$

and boundary conditions

$$
u(a, t)=g_{1}(t), \quad u(b, t)=g_{2}(t), \quad 0 \leq t,
$$

where the material response function $\tau(\cdot)$ is defined by

$$
\tau(\kappa) \equiv \alpha_{1}\left(3+\kappa^{2}, 3+\kappa^{2}\right)-\alpha_{-1}\left(3+\kappa^{2}, 3+\kappa^{2}\right),
$$

and where $f_{1}(\cdot), f_{2}(\cdot), g_{1}(\cdot)$ and $g_{2}(\cdot)$ are required to meet the first-order compatibility conditions

$$
f_{1}(a)=g_{1}(0), \quad f_{1}(b)=g_{2}(0), \quad f_{2}(a)=g_{1}^{\prime}(0), \quad f_{2}(b)=g_{2}^{\prime}(0) .
$$

(ii) Gyratory shearing of an annular tube. This motion is of the form

$$
r(R, \Theta, Z, t)=R, \quad \theta(R, \Theta, Z, t)=\Theta+w(R, t), \quad z(R, \Theta, Z, t)=Z,
$$

and the corresponding left Cauchy-Green tensor, in physical components, is found to be

$$
\mathbf{B}=\left(\begin{array}{ccc}
1 & r w_{r} & 0 \\
r w_{r} & 1+\left(r w_{r}\right)^{2} & 0 \\
0 & 0 & 1
\end{array}\right), \quad w_{r} \equiv \partial w / \partial r
$$

Since this tensor clearly has unit determinant, and thus corresponds to an isochoric motion, it follows that the associated stress tensor is given, in physical components, by

$$
\begin{array}{r}
\mathbf{T}=-p\left(\begin{array}{lll}
1 & 0 & 0 \\
0 & 1 & 0 \\
0 & 0 & 1
\end{array}\right)+\alpha_{1}(I, I I) \\
\begin{array}{r}
r w_{r} \\
0
\end{array}\left(\begin{array}{cc}
r w_{r} & 0 \\
1+\left(r w_{r}\right)^{2} & 0 \\
0 & 1
\end{array}\right) \\
+\alpha_{-1}(I, I I)\left(\begin{array}{ccc}
1+\left(r w_{r}\right)^{2} & -r w_{r} & 0 \\
-r w_{r} & 1 & 0 \\
0 & 0 & 1
\end{array}\right),
\end{array}
$$

where

$$
I=I I=3+\left(r w_{r}\right)^{2} .
$$


With the assumption that $p$ is a function only of $r$ and $t,(2.1)$ consequently reduces to

$$
\begin{aligned}
\frac{\partial}{\partial r} p(r, t)= & \frac{\partial}{\partial r}\left\{\alpha_{1}(I, I I)+\left[1+\left(r w_{r}\right)^{2}\right] \alpha_{-1}(I, I I)\right\}+\rho r\left(w_{t}\right)^{2} \\
& +\left(r w_{r}\right)^{2}\left[\alpha_{1}(I, I I)-\alpha_{-1}(I, I I)\right], \\
\rho r \frac{\partial^{2} w}{\partial t^{2}}= & \frac{1}{r^{2}} \frac{\partial}{\partial r}\left\{r^{3}\left[\alpha_{1}(I, I I)-\alpha_{-1}(I, I I)\right] \frac{\partial w}{\partial r} \mid\right.
\end{aligned}
$$

Thus, while the second of these equations may be used to find $w(r, t)$, the first enables us to determine the pressure $p$ which is needed to maintain the motion. In particular, in the annular cross-section of the sheared tube we pose the following initial boundary-value problem for $w$ :

$$
\rho \frac{\partial^{2} w}{\partial t^{2}}(r, t)=\frac{1}{r^{3}} \frac{\partial}{\partial r}\left|r^{3} \tau\left(r w_{r}\right) \frac{\partial w \mid}{\partial r}\right|, \quad 0<a \leq r \leq b, \quad 0 \leq t,
$$

with initial conditions

$$
w(r, 0)=l_{1}(r), \quad w_{t}(r, 0)=l_{2}(r), \quad a \leq r \leq b,
$$

and boundary conditions

$$
w(a, t)=m_{1}(t), \quad w(b, t)=m_{2}(t), \quad 0 \leq t,
$$

where the function $\tau(\cdot)$ was defined by $(2.11)$ and where the functions $l_{1}(\cdot), l_{2}(\cdot)$, $m_{1}(\cdot)$ and $m_{2}(\cdot)$ are required to satisfy the first-order compatibility conditions

$$
l_{1}(a)=m_{1}(0), \quad l_{1}(b)=m_{2}(0), \quad l_{2}(a)=m_{1}^{\prime}(0), \quad l_{2}(b)=m_{2}^{\prime}(0) .
$$

Wang [17] has studied motions of the above types for one of the simplest theories of finite elasticity; that of the Mooney-Rivlin material for which $\alpha_{1}$ and $\alpha_{-1}$ and, therefore, $\tau$ are constants. In this case, (2.8) and (2.18) become linear, and so Wang was able to find explicit solutions in Fourier-Bessel series form for the free-vibration cases which he considered. In our analysis we exclude the degenerate case studied by Wang, and concern ourselves only with materials such that $\tau(\kappa)$ has nontrivial dependence on $\kappa$. It is convenient to introduce the notation

$$
\Phi(\kappa) \equiv\left\{[\kappa \tau(\kappa)]^{\prime}\right\}^{1 / 2}
$$

We shall require $\Phi^{2}(\cdot)$ to be non-constant and strictly positive for all arguments. An admissible graph for the function $\Phi(\cdot)$ is given in Fig. 1 ; since $\Phi(\cdot)$ is an even function, only the domain of positive arguments is shown in the figure. Physically, the requirement that $\Phi^{2}(\cdot)$ be strictly positive is equivalent to the condition that the shear stress is an increasing function of shear. In mathematical terms, this positivity restriction corresponds to the condition that the dynamical equations (2.8) and (2.18) are strictly hyperbolic.

We devote the remainder of this section to reformulating the above problems in terms 


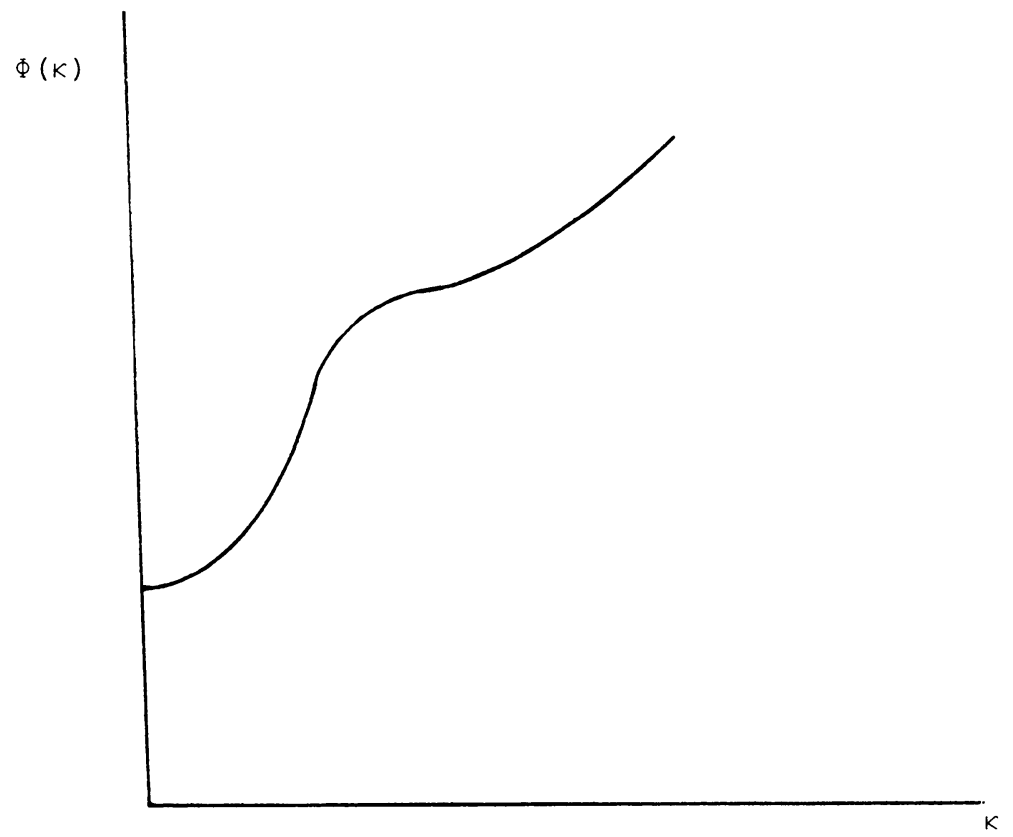

FIG. 1. An admissible graph of the material function $\Phi(\cdot)$.

of Riemann invariants. Each of the above dynamical equations, when rewritten as a first-order system, has the form

$$
\frac{\partial}{\partial t}\left(\begin{array}{l}
u_{1} \\
u_{2}
\end{array}\right)+\left(\begin{array}{cr}
0 & -1 \\
-\frac{1}{\rho}\left[u_{1} \tau\left(u_{1}\right)\right]^{\prime} & 0
\end{array}\right) \frac{\partial}{\partial r}\left(\begin{array}{l}
u_{1} \\
u_{2}
\end{array}\right)=\frac{1}{r}\left(\begin{array}{l}
k_{1}\left(u_{1}, u_{2}\right) \\
k_{2}\left(u_{1}, u_{2}\right)
\end{array}\right),
$$

where, for longitudinal shearing, the functions $u_{i}, k_{i}, i=1,2$, are given by

$$
u_{1} \equiv \frac{\partial u}{\partial r}, \quad u_{2} \equiv \frac{\partial u}{\partial t}, \quad k_{1} \equiv 0, \quad k_{2} \equiv u_{1} \tau\left(u_{1}\right) / \rho,
$$

while for gyratory shearing we have

$$
u_{1} \equiv r \frac{\partial w}{\partial r}, \quad u_{2} \equiv r \frac{\partial w}{\partial t}, \quad k_{1} \equiv-u_{2}, \quad k_{2} \equiv 2 u_{1} \tau\left(u_{1}\right) / \rho .
$$

For the system (2.23) we introduce Riemann invariants $y$ and $z$ (not to be confused with our earlier use of $z$ as a coordinate variable) in the usual way as follows:

$$
y \equiv u_{2}+M\left(u_{1}\right), z \equiv u_{2}-M\left(u_{1}\right)
$$

where

$$
M\left(u_{1}\right) \equiv \rho^{-1 / 2} \int_{0}^{u_{1}} \Phi(\kappa) d \kappa,
$$


and where our earlier assumption that $\Phi^{2}(\cdot)$ be strictly positive renders $M(\cdot)$ invertible. In terms of these Riemann invariants the system (2.23) may now be rewritten in the form

$$
\begin{aligned}
& \frac{\partial y}{\partial t}-\bar{\Gamma}(y-z) \frac{\partial y}{\partial r}=\frac{1}{r}\left\{\bar{\Psi}_{2}(y, z)+\Gamma(y-z) \bar{\Psi}_{1}(y, z)\right\}, \\
& \frac{\partial z}{\partial t}+\Gamma(y-z) \frac{\partial z}{\partial r}=\frac{1}{r}\left\{\bar{\Psi}_{2}(y, z)-\bar{\Gamma}(y-z) \bar{\Psi}_{1}(y, z)\right\},
\end{aligned}
$$

where

$$
\begin{gathered}
\Gamma(y-z) \equiv \rho^{-1 / 2} \Phi\left(M^{-1}\left(\frac{1}{2}(y-z)\right)\right), \\
\Psi_{i}(y, z) \equiv k_{i}\left(M^{-1}\left(\frac{1}{2}(y-z)\right), \frac{1}{2}(y+z)\right), \quad i=1,2 .
\end{gathered}
$$

3. The analysis of breakdown of smoothness. We are now nearly in a position to state and prove our main result. As we have seen, the dynamical equations for our two cases take very similar forms when expressed in terms of Riemann invariants. We shall therefore confine our detailed discussion to the case of gyratory shearing, and subsequently indicate how an almost identical analysis may be carried out in the slightly simpler case of longitudinal shearing.

For gyratory shearing, our initial boundary-value problem, in Riemann invariant form, is readily seen, with the aid of (2.25)-(2.29), to have the form

$$
\begin{gathered}
\frac{\partial y}{\partial t}-\Gamma(y-z) \frac{\partial y}{\partial r}=\frac{1}{r}\left\{2 \Gamma(y-z)+\frac{1}{2}(y+z) \Gamma(y-z)\right\}, \\
\frac{\partial z}{\partial t}+\bar{\Gamma}(y-z) \frac{\partial z}{\partial r}=\frac{1}{r}\left\{2 \Lambda(y-z)-\frac{1}{2}(y+z) \bar{\Gamma}(y-z)\right\}, \\
y(r, 0)=y_{0}(r), \quad z(r, 0)=z_{0}(r), \quad a \leq r \leq b, \\
y(a, t)+z(a, t)=h_{1}(t), \quad y(b, t)+z(b, t)=h_{2}(t), \quad 0 \leq t,
\end{gathered}
$$

where

$$
\begin{aligned}
h_{1}(t) & \equiv 2 a m_{1}^{\prime}(t), \quad h_{2}(t) \equiv 2 b m_{2}^{\prime}(t), \\
\bar{\Lambda}(y-z) & \equiv M^{-1}\left(\frac{1}{2}(y-z)\right) \tau\left(M^{-1}\left(\frac{1}{2}(y-z)\right)\right) .
\end{aligned}
$$

In the following, we shall assume that the function $\tau(\cdot)$ is twice continuously differentiable, so that $\overrightarrow{\Gamma(\cdot)}$ is continuously differentiable. In addition, we have the relation

$$
\Gamma^{\prime}(s)=\frac{[\kappa \tau(\kappa)]^{\prime \prime}}{4[\kappa \tau(\kappa)]^{\prime}},
$$

where

$$
s \equiv 2 M(\kappa)=y-z .
$$

Thus, if $\kappa_{0}$ is such that $\left[\kappa_{0} \tau\left(\kappa_{0}\right)\right]^{\prime \prime}>0$, there exist positive constants $\varepsilon$ and $\delta$ such that

$$
\Gamma^{\prime}(s) \geq \varepsilon>0, \forall\left|s-s_{0}\right| \leq \delta,
$$

where

$$
s_{0} \equiv 2 M\left(\kappa_{0}\right)
$$


Since $\tau(\cdot)$ is a non-constant even function, there will exist infinitely many such points $\kappa_{0}$.

We now establish some helpful preliminary definitions and notations. Let $r_{1}(t)$ and $r_{2}(t)$ respectively denote $y$ - and $z$-characteristic curves of the system (3.1) which emanate from the $r$-axis. Then $r_{1}(\cdot)$ and $r_{2}(\cdot)$ must satisfy the relations

$$
\begin{aligned}
& r_{1}(t)=r_{1}(0)-\int_{0}^{t} \Gamma\left(y_{1}(\tau)-z_{1}(\tau)\right) d \tau, \\
& r_{2}(t)=r_{2}(0)+\int_{0}^{t} \Gamma\left(y_{2}(\tau)-z_{2}(\tau)\right) d \tau,
\end{aligned}
$$

where we have adopted the compact notation

$$
y_{i}(t) \equiv y\left(r_{i}(t), t\right), \quad z_{i}(t) \equiv z\left(r_{i}(t), t\right), \quad i=1,2,
$$

and where, along their associated characteristics, $y$ and $z$ must satisfy, respectively,

$$
\begin{aligned}
y_{1}(t)= & y_{0}\left(r_{1}(0)\right)+\int_{0}^{t} \frac{1}{r_{1}(\tau)}\left\{2 \Lambda\left(y_{1}(\tau)-z_{1}(\tau)\right)\right. \\
& \left.+\frac{1}{2}\left(y_{1}(\tau)+z_{1}(\tau)\right) \Gamma\left(y_{1}(\tau)-z_{1}(\tau)\right)\right\} d \tau \\
z_{2}(t)= & z_{0}\left(r_{2}(0)\right)+\int_{0}^{t} \frac{1}{r_{2}(\tau)}\left\{2 \Lambda\left(y_{2}(\tau)-z_{2}(\tau)\right)\right. \\
& \left.-\frac{1}{2}\left(y_{2}(\tau)+z_{2}(\tau)\right) \Gamma\left(y_{2}(\tau)-z_{2}(\tau)\right)\right\} d \tau
\end{aligned}
$$

It remains to obtain integral expressions of this type for $y_{2}(t)$ and $z_{1}(t)$. To this end. suppose we momentarily fix $t$ and let $r_{1 ; 2}(\tau ; t), \tau \geq 0$, denote that ty-characteristic which intersects the $z$-characteristic $r_{2}(\cdot)$ at the point $\left(r_{2}(t), t\right)$, (i.e., $\left.r_{1 ; 2}(t, t)=r_{2}(t)\right)$, and which meets the $r$-axis at $r_{1 ; 2}(0 ; t)$. Then by virtue of $(3.5)_{1}$ we may write

$$
r_{2}(t)=r_{1 ; 2}(0 ; t)-\int_{0}^{t} \Gamma\left(y_{1 ; 2}(\tau ; t)-z_{1 ; 2}(\tau ; t)\right) d \tau
$$

where

$$
y_{1 ; 2}(\tau ; t) \equiv y\left(r_{1 ; 2}(\tau ; t), \tau\right), \quad z_{1 ; 2}(\tau, t) \equiv z\left(r_{1 ; 2}(\tau ; t), \tau\right)
$$

and where, by $(3.6)_{1}$ and because $y_{1 ; 2}(t ; t)=y_{2}(t)$, we have

$$
\begin{aligned}
y_{2}(t)= & y_{0}\left(r_{1 ; 2}(0 ; t)\right)+\int_{0}^{t} \frac{1}{r_{1 ; 2}(\tau ; t)}\left\{2 \Lambda\left(y_{1 ; 2}(\tau ; t)-z_{1 ; 2}(\tau ; t)\right)\right. \\
& \left.+\frac{1}{2}\left(y_{1 ; 2}(\tau ; t)+z_{1 ; 2}(\tau ; t)\right) \Gamma\left(y_{1 ; 2}(\tau ; t)-z_{1 ; 2}(\tau ; t)\right)\right\} d \tau .
\end{aligned}
$$

An integral expression for $z_{1}(t)$ may be obtained in a completely analogous way. The relation between the various characteristic curves is illustrated in Fig. 2.

We note that the relations (3.5)-(3.8) hold for arbitrary $y$-and $z$-characteristics, and that in the analysis which follows we shall consider two different $z$-characteristics. As above, it is convenient to denote those quantities which are associated with that $z$ characteristic which passes through $\left(r_{2}(0), 0\right)$, as illustrated in Fig. 2, by

$$
r_{i}(\cdot), y_{i}(\cdot), z_{i}(\cdot), i=1,2, \quad r_{1 ; 2}(\cdot ; \cdot), y_{1 ; 2}(\cdot ; \cdot), z_{1 ; 2}(\cdot ; \cdot) \text {, }
$$




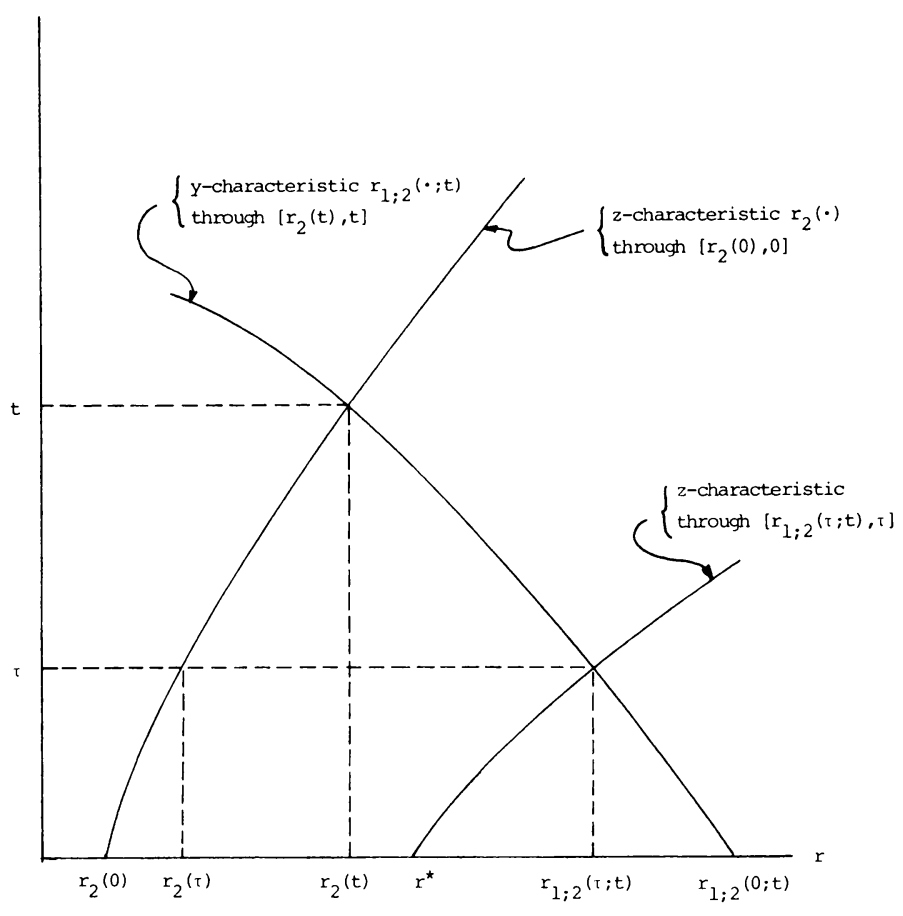

FIG. 2. Characteristic curves at fixed time $t$.

and we shall distinguish the corresponding quantities which are associated with a second $z$-characteristic, which passes through $\left(\tilde{r}_{2}(0), 0\right)$, say, by means of a tilde superscript, i.e.,

$$
\tilde{r}_{i}(\cdot), \tilde{y}_{i}(\cdot), \tilde{z}_{i}(\cdot), i=1,2, \quad \tilde{r}_{1: 2}(\cdot ; \cdot), \tilde{y}_{1 ; 2}(\cdot ; \cdot), \tilde{z}_{1 ; 2}(\cdot ; \cdot) \text {. }
$$

Rozhdestvenskii's [12] method of analysis is to study two adjacent characteristics which belong to the same family, and to impose certain reasonable conditions on the initial data so that they will not only intersect, but also so that along these characteristics the solution tends to different limits at the point of intersection. With this as motivation, and in terms of the above preliminary considerations, we now state and prove our main result: Let $\kappa_{0}, s_{0}, \delta$ and $\varepsilon$ be as in (3.4). Let $y_{0}(\cdot):[a, b] \rightarrow \mathbb{R}$ be an arbitrary $C^{\infty}$ function, such that

$$
\max _{a \leq r \leq b}\left|y_{0}^{\prime}(r)\right| \leq \frac{\delta}{24(b-a)} .
$$

Then there exists smooth initial data $z_{0}(\cdot):[a, b] \rightarrow \mathbb{R}$ such that the smooth solution of $(3.1)$ will exist only for a finite time.

In the proof of this theorem we shall make use of the following definitions:

$$
\begin{gathered}
K \equiv \max _{a \leq r \leq b}\left|y_{0}(r)\right|, \\
M_{1} \equiv \max _{y, z \in G_{0}}\left\{2|\bar{\Lambda}(y-z)|+\frac{1}{2}|y+z| \bar{\Gamma}(y-z)\right\}, \\
M_{2} \equiv \max _{\left|y-z-s_{0}\right| \leq \delta} \bar{\Gamma}(y-z),
\end{gathered}
$$


where the set $G_{0}$ is given by

$$
G_{0} \equiv\left\{(y, z)|| y-z-s_{0}|\leq \delta,| y+z|\leq 2 K+| s_{0} \mid+4 \delta\right\} .
$$

Now, choose points $r_{2}(0), r_{2}(0)+\alpha$ in the interval $(a, b)$, where $\alpha$ is some positive constant yet to be determined and subject at this stage only to the requirement that

$$
r_{2}(0)+(1+\gamma) \alpha<b
$$

where

$$
\gamma \equiv 50 M_{2} / \delta \varepsilon
$$

In addition, assign a value of $z_{0}\left(r_{2}(0)\right)$ which gives

$$
\left|y_{0}\left(r_{2}(0)\right)-z_{0}\left(r_{2}(0)\right)-s_{0}\right| \leq \delta / 6
$$

and put

$$
z_{0}\left(r_{2}(0)+\alpha\right)=z_{0}\left(r_{2}(0)\right)+\frac{\delta}{12} .
$$

Let $z_{0}(\cdot):[a, b] \rightarrow \mathbb{R}$ be any $C^{\infty}$ function which satisfies (3.15), (3.16) and the additional requirement that

$$
\left|y_{0}(r)-z_{0}(r)-s_{0}\right| \leq \delta / 3, \quad r_{2}(0) \leq r \leq r_{2}(0)+(1+\gamma) \alpha .
$$

In accordance with the notation defined earlier, let $\tilde{r}_{2}(t)$ be the $z$-characteristic which emanates from the point $\left(r_{2}(0)+\alpha, 0\right)$.

Now, from what already has been developed above, it is straightforward to obtain the following inequalities:

$$
\begin{gathered}
z_{0}\left(r_{2}(0)\right)-\frac{1}{a} M_{1} t \leq z_{2}(t) \leq z_{0}\left(r_{2}(0)\right)+\frac{1}{a} M_{1} t \\
z_{0}\left(r_{2}(0)\right)+\frac{\delta}{12}-\frac{1}{a} M_{1} t \leq \tilde{z}_{2}(t) \leq z_{0}\left(r_{2}(0)\right)+\frac{\delta}{12}+\frac{1}{a} M_{1} t \\
y_{0}\left(r_{1 ; 2}(0, t)\right)-\frac{1}{a} M_{1} t \leq y_{2}(t) \leq y_{0}\left(r_{1 ; 2}(0 ; t)\right)+\frac{1}{a} M_{1} t \\
y_{0}\left(\tilde{r}_{1 ; 2}(0 ; t)\right)-\frac{1}{a} M_{1} t \leq \tilde{y}_{2}(t) \leq y_{0}\left(\tilde{r}_{1 ; 2}(0 ; t)\right)+\frac{1}{a} M_{1} t
\end{gathered}
$$

for all $t$ such that

$$
\begin{gathered}
\left(y_{2}(\xi), z_{2}(\xi)\right) \in G_{0}, \quad\left(\tilde{y}_{2}(\xi), \tilde{z}_{2}(\xi)\right) \in G_{0}, \\
\left(y_{1 ; 2}(\tau ; \xi), z_{1 ; 2}(\tau ; \xi)\right) \in G_{0}, \quad\left(\tilde{y}_{1 ; 2}(\tau ; \xi), \tilde{z}_{1 ; 2}(\tau ; \xi)\right) \in G_{0}, \quad 0 \leq \tau \leq \xi \leq t .
\end{gathered}
$$

Now, with the aid of the mean value theorem and in accordance with $(3.5)_{2}$ and its counterpart for $\tilde{r}_{2}(t)$, we may write

$$
\tilde{r}_{2}(t)-r_{2}(t)=\alpha+\int_{0}^{t} \bar{\Gamma}^{\prime}(\phi(\tau))\left[\tilde{y}_{2}(\tau)-y_{2}(\tau)+z_{2}(\tau)-\tilde{z}_{2}(\tau)\right] d \tau
$$


where $\phi(\tau)$ is a number intermediate between $y_{2}(\tau)-z_{2}(\tau)$ and $\tilde{y}_{2}(\tau)-\tilde{z}_{2}(\tau)$ for each $0 \leq \tau \leq t$. In addition, it follows from the inequalities (3.17) and (3.18), and the results of $(3.4)_{1},(3.12)$, and $(3.19)_{1,2}$, that

$$
\begin{aligned}
\tilde{y}_{2}(\tau)-y_{2}(\tau)+z_{2}(\tau)-\tilde{z}_{2}(\tau) & \leq \frac{4}{a} M_{1} \tau-\frac{\delta}{24}, \\
\Gamma^{\prime}(\phi(\tau)) \geq \varepsilon>0, \quad 0 & \leq \tau \leq t .
\end{aligned}
$$

Thus, we have

$$
\tilde{r}_{2}(t)-r_{2}(t) \leq \alpha+\varepsilon \int_{0}^{t}\left[\frac{4}{a} M_{1} \tau-\frac{\delta}{24}\right] d \tau=\frac{2 \varepsilon M_{1}}{a} Q(t),
$$

where

$$
Q(t) \equiv t^{2}-\frac{a \delta}{48 M_{1}} t+\frac{a \alpha}{2 \varepsilon M_{1}} .
$$

Note that the roots of $Q(t)=0$ are given by

$$
t_{ \pm}=\frac{a \delta}{96 M_{1}} \pm\left\{\left(\frac{a \delta}{96 M_{1}}\right)^{2}-\frac{a \alpha}{2 \varepsilon M_{1}}\right\}^{1 / 2}
$$

and are real for $0 \leq \alpha \leq\left(2 a \varepsilon / M_{1}\right)(\delta / 96)^{2}$.

We now seek a value $t_{c}$ for the time $t$ such that conditions (3.19) are necessarily satisfied, and such that for $0 \leq t \leq t_{c}$,

$$
r_{1 ; 2}(0 ; t) \leq r_{2}(0)+(1+\gamma) \alpha, \quad \tilde{r}_{1 ; 2}(0 ; t) \leq r_{2}(0)+(1+\gamma) \alpha .
$$

Toward this end, we first suppose that all of the conditions (3.19), (3.25) are met for $0 \leq t \leq t^{*}$ and that at least one of them is satisfied extremally at the time $t=t^{*}$. This necessitates the separate consideration of the ten possible cases listed below, in each of which we shall obtain a positive lower bound for $t^{*}$; ultimately we choose a positive $t_{c}$ which is less than the least of these ten values. To enumerate these ten possibilities, suppose that for each case the conditions (3.19) and (3.25) are satisfied for $0 \leq t \leq t_{i}^{*}$, $i=1,2, \ldots, 10$, respectively, and that correspondingly

(i) $r_{1 ; 2}\left(0 ; t_{1}^{*}\right)=r_{2}(0)+(1+\gamma) \alpha$,

(ii) $\tilde{r}_{1 ; 2}\left(0 ; t_{2}^{*}\right)=r_{2}(0)+(1+\gamma) \alpha$,

(iii) $\left|y_{2}\left(t_{3}^{*}\right)-z_{2}\left(t_{3}^{*}\right)-s_{0}\right|=\delta$,

(iv) $\left|\tilde{y}_{2}\left(t_{4}^{*}\right)-\tilde{z}_{2}\left(t_{4}^{*}\right)-s_{0}\right|=\delta$,

(v) $\left|y_{1 ; 2}\left(\tau_{5}^{*} ; t_{5}^{*}\right)-z_{1 ; 2}\left(\tau_{5}^{*}, t_{5}^{*}\right)-s_{0}\right|=\delta$

for some $\tau_{5}^{*} \in\left[0, t_{5}^{*}\right]$,

(vi) $\left|\tilde{y}_{1 ; 2}\left(\tau_{6}^{*} ; t_{6}^{*}\right)-\tilde{z}_{1 ; 2}\left(\tau_{6}^{*} ; t_{6}^{*}\right)-s_{0}\right|=\delta$

for some $\tau_{5}^{*} \in\left[0, t_{5}^{*}\right]$,

(vii) $\left|y_{2}\left(t_{7}^{*}\right)+z_{2}\left(t_{7}^{*}\right)\right|=2 K+\left|s_{0}\right|+4 \delta$,

(viii) $\left|\tilde{y}_{2}\left(t_{8}^{*}\right)+\tilde{z}_{2}\left(t_{8}^{*}\right)\right|=2 K+\left|s_{0}\right|+4 \delta$, 
(ix) $\left|y_{1 ; 2}\left(\tau_{9}^{*} ; t_{9}^{*}\right)+z_{1 ; 2}\left(\tau_{9}^{*} ; t_{9}^{*}\right)\right|=2 K+\left|s_{0}\right|+4 \delta$

for some $\tau_{9}^{*} \in\left[0, t_{9}^{*}\right]$,

(x) $\left|\tilde{y}_{1 ; 2}\left(\tau_{10}^{*} ; t_{10}^{*}\right)+\tilde{z}_{1 ; 2}\left(\tau_{10}^{*} ; t_{10}^{*}\right)\right|=2 K+\left|s_{0}\right|+4 \delta$

for some $\tau_{10}^{*} \in\left[0, t_{10}^{*}\right]$.

Consider now the similar cases (i) and (ii). In particular, in case (ii) we have from (3.5), (3.7), and (3.9) $)_{2}$, that

$$
\begin{aligned}
r_{2}(0)+ & (1+\gamma) \alpha-\int_{0}^{t_{2}^{*}} \Gamma\left(\tilde{y}_{1 ; 2}\left(\tau ; t_{2}^{*}\right)-\tilde{z}_{1 ; 2}\left(\tau ; t_{2}^{*}\right)\right) d \tau \\
& =r_{2}(0)+\alpha+\int_{0}^{t_{2}^{*}} \Gamma\left(\tilde{y}_{2}(\tau)-\tilde{z}_{2}(\tau)\right) d \tau,
\end{aligned}
$$

which, by virtue of inequalities (3.18), yields

$$
t_{2}^{*} \geq \gamma \alpha / 2 M_{2} \text {. }
$$

An identical analysis for the case (i) gives

$$
t_{1}^{*} \geq(1+\gamma) \alpha / 2 M_{2} .
$$

Cases (iii) and (iv) may be treated similarly and for the latter it follows from (3.10), (3.15), (3.16) and (3.18), that

$$
\begin{aligned}
\delta= & \left|\tilde{y}_{2}\left(t_{4}^{*}\right)-\tilde{z}_{2}\left(t_{4}^{*}\right)-s_{0}\right| \leq\left|\tilde{y}_{2}\left(t_{4}^{*}\right)-y_{0}\left(\tilde{r}_{1 ; 2}\left(0 ; t_{4}^{*}\right)\right)\right| \\
& +\left|\tilde{z}_{2}\left(t_{4}^{*}\right)-z_{0}\left(r_{2}(0)\right)-\frac{\delta}{12}\right|+\frac{\delta}{12}+\left|y_{0}\left(\tilde{r}_{1 ; 2}\left(0 ; t_{4}^{*}\right)\right)-y_{0}\left(r_{2}(0)\right)\right| \\
& +\left|y_{0}\left(r_{2}(0)\right)-z_{0}\left(r_{2}(0)\right)-s_{0}\right| \leq \frac{2}{a} M_{1} t_{4}^{*}+\frac{7 \delta}{24}
\end{aligned}
$$

Thus, we have

$$
t_{4}^{*} \geq 17 a \delta / 48 M_{1},
$$

and analogously for case (iii) we find

$$
t_{3}^{*} \geq 19 a \delta / 48 M_{1} \text {. }
$$

Cases (v) and (vi) admit to a similar analysis, and for (v) we see, using (3.5), (3.8), (3.15), (3.17) and (3.18) that

$$
\begin{aligned}
\delta= & \left|y_{1 ; 2}\left(\tau_{5}^{*} ; t_{5}^{*}\right)-z_{1 ; 2}\left(\tau_{5}^{*}, t_{5}^{*}\right)-s_{0}\right| \leq\left|y_{1 ; 2}\left(\tau_{5}^{*} ; t_{5}^{*}\right)-y_{1 ; 2}\left(0 ; t_{5}^{*}\right)\right| \\
& +\left|z_{1 ; 2}\left(\tau_{5}^{*} ; t_{5}^{*}\right)-z_{0}\left(r^{*}\right)\right|+\left|y_{0}\left(r^{*}\right)-y_{0}\left(r_{1 ; 2}\left(0 ; t_{5}^{*}\right)\right)\right| \\
& +\left|y_{0}\left(r^{*}\right)-z_{0}\left(r^{*}\right)-s_{0}\right| \leq \frac{\delta}{3}+\frac{\delta}{24}+\frac{2}{a} M_{1} t_{5}^{*},
\end{aligned}
$$

which yields

$$
t_{5}^{*} \geq 5 a \delta / 16 M_{1}
$$


Here, $r^{*}$ corresponds to that value on the $r$-axis which is joined to the point $\left(r_{1: 2}\left(\tau_{5}^{*} ; t_{5}^{*}\right)\right.$, $\tau_{5}^{*}$ ) by a $z$-characteristic; it is clear (see Fig. 2 ) that

$$
r_{2}(0)<r^{*}<r_{2}(0)+(1+\gamma) \alpha .
$$

Similarly, for case (vi) we obtain

$$
t_{6}^{*} \geq 5 a \delta / 16 M_{1}
$$

Finally, cases (vii) $-(\mathrm{x})$ admit to similar considerations, and for $(\mathrm{x})$ we see using $(3.9)_{2}$, (3.10), (3.11), (3.18), and (3.19) that

$$
\begin{aligned}
2 K+\left|s_{0}\right|+4 \delta= & \left|\tilde{y}_{1 ; 2}\left(\tau_{10}^{*}, t_{10}^{*}\right)+\tilde{z}_{1 ; 2}\left(\tau_{10}^{*} ; t_{10}^{*}\right)\right| \\
\leq & \left|\tilde{y}_{1 ; 2}\left(\tau_{10}^{*} ; t_{10}^{*}\right)-\tilde{z}_{1 ; 2}\left(\tau_{10}^{*} ; t_{10}^{*}\right)-s_{0}\right|+\left|s_{0}\right| \\
& +2 \mid y_{0}\left(\tilde{r}_{1 ; 2}\left(0 ; t_{10}^{*}\right) \mid+\frac{2}{a} M_{1} t_{10}^{*}\right. \\
\leq & 2 K+\left|s_{0}\right|+\delta+\frac{2}{a} M_{1} t_{10}^{*} .
\end{aligned}
$$

Thus, it follows that

$$
t_{10}^{*} \geq 3 a \delta / 2 M_{1}
$$

and, in a completely analogous way we also see that

$$
t_{k}^{*} \geq 3 a \delta / 2 M_{1}, \quad k=7,8,9 .
$$

In summary, then, it follows that we may take

$$
t_{c} \equiv \min \left\{\frac{\gamma \alpha}{2 M_{2}}, \frac{5 a \delta}{16 M_{1}} \mid\right.
$$

and be assured not only that (3.25) holds for $0 \leq t \leq t_{c}$, but also that (3.19) is satisfied for $0 \leq \tau \leq \xi \leq t_{c}$.

Now, suppose there exists an $\alpha>0$ such that the ordering $0<t_{-}<t_{c}$ can be accomplished, where $t_{-}$is given in (3.24). Then, from (3.18) and (3.24) we see that for $0 \leq t \leq t_{-}$,

$$
\tilde{z}_{2}(t)-z_{2}(t) \geq \frac{\delta}{12}-\frac{2}{a} M_{1} t \geq \frac{\delta}{12}-\frac{2}{a} M_{1} t_{-} \geq \frac{\delta}{12}-\frac{\delta}{48}>0 .
$$

Moreover, from (3.22), (3.24), it follows that there exists a time $t_{0}$ with $0<t_{0} \leq t_{-}$such that

$$
\tilde{r}_{2}\left(t_{0}\right)=r_{2}\left(t_{0}\right)=r_{0},
$$

and this, together with (3.36), yields

$$
\lim _{t \rightarrow t_{0}} z\left(r_{2}(t), t\right) \neq \lim _{t \rightarrow t_{0}} z\left(\tilde{r}_{2}(t), t\right)
$$


which implies that at or before the time $t_{0}$ a smooth solution will cease to exist. Thus, all that remains in order to complete the proof of the theorem is to show that such an $\alpha$ exists. To this end, consider the function

$$
q(\alpha) \equiv \frac{\gamma \alpha}{2 M_{2}}-t_{-}(\alpha)=\frac{25 \alpha}{\delta \varepsilon}-\frac{a \delta}{96 M_{1}}+\left\{\left(\frac{a \delta}{96 M_{1}}\right)^{2}-\frac{a \alpha}{2 \varepsilon M_{1}}\right\}^{1 / 2} .
$$

Clearly, we have

$$
q(0)=0
$$

and

$$
q^{\prime}(0)=\frac{25}{\delta \varepsilon}-\frac{a}{4 \varepsilon M_{1}}\left(\frac{96 M_{1}}{a \delta}\right)=\frac{1}{\delta \varepsilon}>0,
$$

which shows that there exists $\alpha_{*}>0$ such that

$$
t_{-}\left(\alpha_{*}\right)<\gamma \alpha_{*} / 2 M_{2} \text {. }
$$

On the other hand, since for any value of $\alpha(3.24)$ yields

$$
t_{-}(\alpha) \leq a \delta / 96 M_{1}<5 a \delta / 16 M_{1} \text {, }
$$

we see, by comparison with (3.35), that

$$
0<t_{-}\left(\alpha_{*}\right)<t_{c} .
$$

Remark 1. This theorem applies to a rather extensive class of initial data. Our only restriction on $y_{0}(\cdot)$ was that

$$
\max _{a \leq r \leq b}\left|y_{0}^{\prime}(r)\right| \leq \frac{\delta}{24(b-a)},
$$

and the function $z_{0}(\cdot)$ was allowed to be arbitrary outside the interval $\left[r_{2}(0), r_{2}(0)+\right.$ $(1+\gamma) \alpha]$, where $r_{2}(0)$ itself was chosen arbitrarily in $(a, b)$, and where $\alpha$ was assigned any value such that $t_{-}$lies in the interval $\left(0, t_{c}\right)$.

Remark 2. Given $\kappa_{0}, s_{0}, \delta$ and $\varepsilon$ such that (3.4) holds, we could equally well have chosen initial data which would lead to a breakdown of smoothness by inducing $y$ characteristics to intersect, as follows. Let $z_{0}(\cdot)$ be any $C^{\infty}$ function such that $\max _{a \leq r \leq b}\left|z_{0}^{\prime}(r)\right| \leq \delta / 24(b-a)$. Choose a point $r_{1}(0) \in(a, b)$, and let $\alpha$ be any sufficiently small positive constant as in the proof above. Then, assign a value of $y_{0}\left(r_{1}(0)\right)$ such that $\left|y_{0}\left(r_{1}(0)\right)-z_{0}\left(r_{1}(0)\right)-s_{0}\right| \leq \delta / 6$ and set $y_{0}\left(r_{1}(0)+\alpha\right)=y_{0}\left(r_{1}(0)\right)+\delta / 12$. Finally, require that $\left|y_{0}(r)-z_{0}(r)-s_{0}\right| \leq \delta / 3, r_{1}(0) \leq r \leq r_{1}(0)+(1+\gamma) \alpha$, where $\gamma$ is defined by (3.14). With these conditions satisfied, it follows that a sufficiently small choice of $\alpha$ will ensure that $y$-characteristics intersect and that a breakdown of smoothness in the solution will arise.

Remark 3. Suppose, contrary to (3.4), that $\bar{\Gamma}^{\prime}(s) \leq-\varepsilon<0$ for $\left|s-s_{0}\right| \leq \delta$. Then, as above, we still may guarantee the non-existence of a smooth solution for all time by way of intersecting $z$-characteristics. The only change in the analysis as just given is the replacement of $+\delta / 12$ with $-\delta / 12$ in (3.16). Of course, a result similar to that mentioned in Remark 2 also may be derived here. 
Remark 4. Since the equations of longitudinal shearing, in Riemann-invariant form, are given by

$$
\frac{\partial y}{\partial t}-\bar{\Gamma}(y-z) \frac{\partial y}{\partial r}=\frac{1}{r} \bar{\Lambda}(y-z), \quad \frac{\partial z}{\partial t}+\bar{\Gamma}(y-z) \frac{\partial z}{\partial r}=\frac{1}{r} \bar{\Lambda}(y-z),
$$

a simplification of the above proof may be used to demonstrate the non-existence of a global smooth solution. In this case, we may take, instead of $G_{0}$ in (3.12), the set $\left\{(y, z)|| y-z-s_{0} \mid \leq \delta\right\}$.

Remark 5. The above analysis is essentially that of Rozhdestvenskii [12]. He was content, however, to exhibit but one example of initial data which led to a breakdown of smoothness. We on the other hand, have concentrated on finding an extensive class of such data.

Remark 6. The proof of this theorem has revealed that when $\alpha$ is sufficiently small, the root $t_{-}$in (3.24) represents an upper bound on the time at which a smooth solution must cease to exist. Approximately, we have

$$
t_{-} \sim 24 \alpha / \delta \varepsilon=2 / \varepsilon z_{0}^{\prime}(\bar{r})
$$

where $\bar{r}$ is some value intermediate between $r_{2}(0)$ and $r_{2}(0)+\alpha$. For the case in which (1.1) satisfies $\lambda_{y} \leq-m_{1}<0, \mu_{z} \leq-m_{2}<0$, analogous to that which we have studied in detail, Lax [4] has obtained an estimate for the critical time to breakdown of the form

$$
t_{\text {Lax }} \sim C / m_{2} q_{0},
$$

where $q_{0}=z_{x}\left(x_{0}, 0\right)$ and $C$ is a constant which depends on the initial data. An estimate of this kind also has been given by Jeffrey [9] and is implicit in the work of Slemrod [11]. While the estimate (3.42) as well as those of Lax, Jeffrey, and Slemrod are derived somewhat differently, these various approaches rely on (i) a condition of genuine nonlinearity, and (ii) a certain postulate concerning the sign and the magnitude of the first derivatives of some part of the initial data.

Remark 7. MacCamy and Mizel [7] consider a class of functions $Q(\cdot)$ in (1.2) such that $\bar{\Gamma}^{\prime}(0)=0$. They further choose initial data such that $y_{0}-z_{0} \equiv 0$, and thus it is not feasible to draw analogies between their result and the present work.

\section{REFERENCES}

[1] R. Courant and K. O. Friedrichs, Supersonic flow and shock waves, Wiley-Interscience, New York, 1948

[2] R. Courant and P. D. Lax, Nonlinear partial differential equations with two dependent variables, Comm. Pure Appl. Math. 2 (1949)

[3] P. D. Lax, Hyperbolic systems of conservation laws II, Comm. Pure Appl. Math. 10 (1957)

[4] P. D. Lax, Development of singularities of solutions of nonlinear partial differential equations, J. Mathematical Phys. 5 (1964)

[5] P. D. Lax, Hyperbolic systems of conservation laws and the mathematical theory of shock waves, in Regional Conference Series in Applied Mathematics, SIAM 1973

[6] N. J. Zabusky, Exact solution for the vibrations of a nonlinear continuous model string, J. Mathematical Phys. 3 (1962)

[7] R. C. MacCamy and V. J. Mizel, Existence and non-existence in the large of solutions of quasilinear wave equations, Arch. Rat. Mech. Anal. 25 (1967)

[8] A. Jeffrey, The evolution of discontinuities in solutions of homogeneous nonlinear hyperbolic equations having smooth initial data, J. Math. Mech. 17 (1967) 
[9] A. Jeffrey, Quasilinear hyperbolic systems and waves. Research Notes in Mathematics, Pitman, San Francisco, 1976

[10] J. Nishida, Global smooth solutions for the second-order quasilinear wave equation with the first-order dissipation, unpublished (1975)

[11] M. Slemrod, Instability of steady shearing flows in a nonlinear viscoelastic fluid. Arch. Rat. Mech. Anal. 68 (1979)

[12] B. L. Rozhdestvenskii, On the discontinuity of solutions of quasilinear wave equations, Amer. Math. Soc. Transl. (2) 101 (1973)

[13] F. John, Formation of singularities in one-dimensional nonlinear wave propagation, Comm. Pure Appl. Math. 27 (1974)

[14] W. Kosinski, Gradient catastrophe in the solution of nonlinear hyperbolic systems, J. Math. Anal. Appl. 61 (1977)

[15] P. H. Chang, On the breakdown phenomena of solutions of quasilinear wave equations, Mich. Math. J. 23 (1976)

[16] V. Thomée, Difference methods for two-dimensional mixed problems for hyperbolic first-order systems, Arch. Rat. Mech. Anal. 8 (1961).

[17] A. S. D. Wang, On free oscillations of elastic incompressible bodies in finite shear, Int. J. Engng. Sci. 7 (1969)

[18] C. C. Wang and C. Truesdell, Introduction to rational elasticity, Nordhoff, 1973

[19] R. J. Knops, H. A. Levine and L. E. Payne, Non-existence, instability, and growth theorems for solutions of a class of abstract nonlinear equations with applications to nonlinear elastodynamics, Arch. Rational Mech. Analysis 55 (1974)

[20] D. G. Schaeffer, A regularity theorem for conservation laws, Advances Math. 11 (1973). 